\title{
Mega-parsec scale magnetic fields in low density regions in the SKA era: filaments connecting galaxy clusters and groups
}

\author{
Gabriele Giovannini ${ }^{1,2}$, Annalisa Bonafede ${ }^{3}$, Shea Brown ${ }^{4}$, Luigina Feretti ${ }^{1}$, Chiara \\ Ferrari $^{5}$, Myriam Gitti ${ }^{1,2}$, Federica Govoni ${ }^{6}$, Matteo Murgia ${ }^{6}$, and Valentina Vacca ${ }^{7}$ \\ ${ }^{1}$ Istituto di Radioastronomia/INAF (BO), I; ${ }^{2}$ Dipartimento di Fisica e Astronomia, Bologna \\ University, I; ${ }^{3}$ Hamburger Sternwarte, Universitat, D; ${ }^{4}$ Iowa University, USA; ${ }^{5}$ Lagrange \\ Laboratory, OCA, F; ${ }^{6}$ Osservatorio Astronomico di Cagliari/INAF, I; ${ }^{7}$ Max Planck Institute for \\ Astrophysics, $D$. \\ E-mail: ggiovanneira.inaf.it
}

\begin{abstract}
The presence of magnetic fields in galaxy clusters has been well established in recent years, and their importance for the understanding of the physical processes at work in the Intra Cluster Medium has been recognized. Halo and relic sources have been detected in several tens clusters. A strong correlation is present between the halo and relic radio power and the X-ray luminosity. Since cluster X-Ray luminosity and mass are related, the correlation between the radio power and $\mathrm{X}$-ray luminosity could derive from a physical dependence of the radio power on the cluster mass, therefore the cluster mass could be a crucial parameter in the formation of these sources. The goal of this project is to investigate the existence of non-thermal structures beyond the Mpc scale, and associated with lower density regions with respect to clusters of galaxies: galaxy filaments connecting rich clusters. We present a piece of evidence of diffuse radio emission in intergalactic filaments. Moreover, we present and discuss the detection of radio emission in galaxy groups and in faint X-Ray clusters, to analyze non-thermal properties in low density regions with physical conditions similar to galaxy filaments. We discuss how SKA1 observations will allow the investigation of this topic and the study of the presence of diffuse radio sources in low density regions. This will be a fundamental step to understand the origin and properties of cosmological magnetic fields.
\end{abstract}

Advancing Astrophysics with the Square Kilometre Array

June 8-13, 2014

Giardini Naxos, Sicily, Italy 


\section{Introduction}

Magnetism is one of the four fundamental forces and plays an important role in the formation and evolution of objects as large as clusters of galaxies and as small as stars. In spite of the significance of magnetic fields in astrophysics and cosmology, their origin and properties remain poorly understood. Observational evidences derived primarily during the last decade (see e.g. the recent review by Feretti et al. 2012) show that extended magnetic fields within the Intra Cluster Medium (ICM) are common in galaxy clusters:

- for $\sim 70$ clusters deep radio observations have established the presence of synchrotron extended sources (halos and relics) that are not associated with individual galaxies, but with the ICM as a whole. The observed radio features imply the existence of $\mu \mathrm{G}$ magnetic fields on scales $\sim 1 \mathrm{Mpc}$; - Faraday-rotation measures of polarized radio sources both within and behind clusters represent independent probes of the strength of intracluster magnetic fields. Studies on both statistical samples and individual clusters (see e.g. Bonafede et al. 2013; Vacca et al. 2012) yield consistent values of $1-5 \mu \mathrm{G}$, in cluster central regions.

In addition, evidence has been found for the existence of magnetic fields on even larger scales and in environments of lower density, although in many cases the physical nature of the corresponding structures remains ambiguous.

Here we will present the observational evidence of non-thermal emission in galaxy filaments, poor clusters and low mass regions. These data can improve our knowledge on the origin of cosmological (Mpc scale) magnetic fields. Finally, we will discuss how important the SKA will be for the investigation of this subject.

\section{Beyond Galaxy Clusters}

The most convincing evidence of radio emission from a genuine filament was presented by Bagchi et al. (2002), who found radio emission coincident with the filament of galaxies $\mathrm{ZwCl}$ 2341.1+0000 (see Boschin et al. 2013, for a recent discussion). VLA observations of this field (Giovannini et al. 2010) confirmed the presence of large-scale diffuse emission extending over an area of $\sim 3 \mathrm{Mpc}$ in size. The surface brightness in the faint external regions is $\sim 5 \times 10^{-5}$ $\mathrm{mJy} / \operatorname{arcsec}^{2}$ at $1.4 \mathrm{GHz}$. The structure is polarized on average at about $10 \%$ level.

An additional case of diffuse radio emission possibly associated with a large-scale filament is the Southern component of 0809+39 (Brown \& Rudnick 2009). Its origin is uncertain, though its coincidence with a filament of galaxies at $\mathrm{z} \sim 0.04$ suggests that it could be either synchrotron emission from filamentary large-scale structure or old emission from an extinct radio galaxy.

A complex radio emission has also been recently found in the A3411-A3412 structure (Giovannini et al. 2013, Fig. 1). ROSAT X-ray data show a diffuse thermal emission from A3411 and a more compact emission coincident with A3412. The A3411 emission towards A3412 shows a multiple structure with a few sub-components suggesting the presence of an active merger. Optical data confirm the existence of sub-structures and show an extended filament to the SE of A3412, aligned with the A3411 - A3412 system.

The radio image shows a halo source in A3411 and an extended structure in between A3411A3412 and in the region of the SE filament (see Fig. 1 and Giovannini et al. 2013). The morphology 


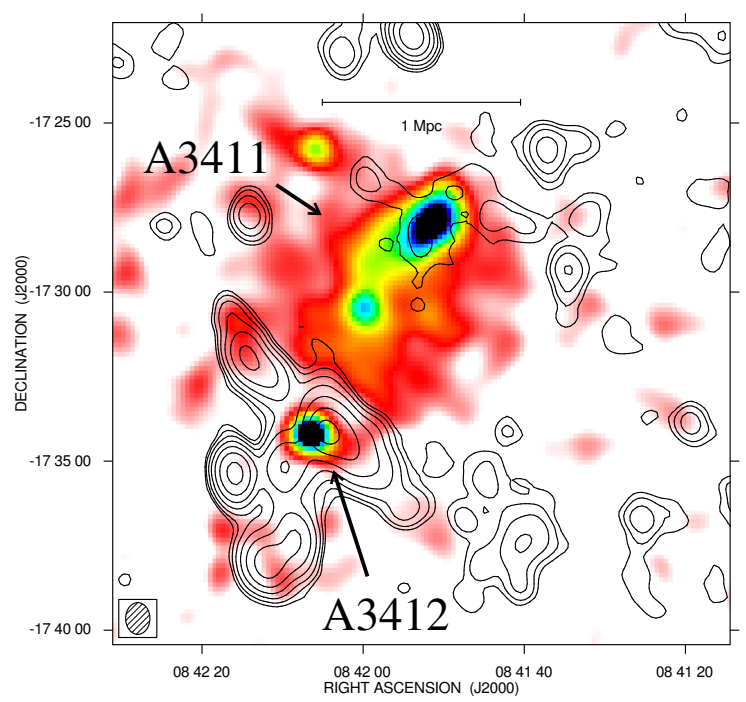

Figure 1: Radio X-ray overlay of the A3411 - A3412 region. Contours show the flux density at $1.4 \mathrm{GHz}$, half power beam width $(\mathrm{HPBW})=56.3$ " $\mathrm{x} 43.1$ ". Contours are from $0.12 \mathrm{mJy} / \mathrm{beam}$ with a step of 0.12 $\mathrm{mJy} / \mathrm{beam}$. In colour the X-ray image from ROSAT data. Note the extended emission aligned with the A3411-A3412 filament. For more details, see Giovannini et al. (2013).

and properties of the diffuse radio emission could be explained by electron acceleration and magnetic field amplification originated from accretion shocks combined with turbulence in the thermal gas due to the merger between A3411 and A3412 clusters and the SE filament. In particular the diffuse source SE to A3412, oriented along the giant filament could be powered by accretion shocks as material falls onto the filament, as it was suggested by Brown \& Rudnick (2011) for the relic of the Coma cluster.

The number of diffuse synchrotron sources connected to galaxy filaments is presently quite low. This is consistent with the relatively high surface brightness limit of present radio surveys (e.g. NVSS) and with the expected low magnetic-field intensity in these regions (e.g. 10 nanoGauss on the volume average for filaments, Ryu et al. 2008). We could speculate that in the few cases where radio emission has been detected from galaxy filaments, peculiar conditions of merging activity among local sub-groups are able to amplify the magnetic fields and accelerate relativistic particles (see e.g. Boschin et al. 2013 for $\mathrm{ZwCl} 2341.1+0000$ and Vazza et al. 2015).

On a smaller scale, bridges of diffuse radio emission have been found in a few clusters connecting radio halos and peripheral relics (Feretti et al. 2012). These structures may trace the presence of synchrotron emission in filaments of merging material into the main cluster. The prototype is the bridge of radio emission connecting the halo Coma-C with the peripheral relic 1253+275 (Kim et al. 1989). This extended low brightness structure was detected in WSRT data and confirmed by low resolution single dish observations. The surface brightness at $90 \mathrm{~cm}$ is lower than $\sim 10^{-4}$ $\mathrm{mJy} / \mathrm{arcsec}^{2}$. Assuming a spectral index of 1.5 , a brightness of $\sim 2 \times 10^{-5} \mathrm{mJy} / \operatorname{arcsec}^{2}$ at $1.4 \mathrm{GHz}$ is derived. At present, we do not know in detail its morphology and properties because of a too low signal to noise ratio in interferometric data and a too low angular resolution in single dish images. This structure is visible also in X-ray images. Its origin is unknown, but we note that it is the same direction of the bridge of galaxies connecting Coma to A1367 and forming the super-cluster 
structure. A large scale magnetic field is present in the Coma cluster peripheral regions (Bonafede et al. 2010, 2013), but the bridge properties are quite different from those of the central halo and of the relic source. Turbulence in the ICM could be invoked as the origin of the radio emission, but this is problematic because turbulence is not efficient in accelerating particles in low density regions, and a connection to shocks is not clear. Similar features have been found in A2255 and A2744 (Feretti et al. 2012).

\section{Poor clusters and low density regions}

Since cluster X-Ray luminosity and mass are correlated, the correlation between radio power and X-ray luminosity in radio halos could reflect a dependence of the radio power on the cluster mass. This correlation could indicate that the cluster mass is a crucial parameter in the formation of radio halos and relics. Since it is likely that massive clusters are the result of several major mergers, we can conclude that the cluster total mass and past plus present mergers are the necessary ingredients for the formation and evolution of diffuse radio sources, in agreement with the result that not all clusters with recent mergers show a radio halo or a relic source.

This scenario is presently supported by many observational and theroretical results and it is also in agreement with numerical simulations (see e.g. Brunetti \& Lazarian 2011, Vazza et al. 2010, Cassano et al. 2010). However, Brown \& Rudnick (2009, 2011), and Giovannini e al. (2009, 2011) have shown the presence of diffuse radio sources in a few clusters with low X-ray luminosity, therefore low mass. Since low-mass clusters and groups are often found in filaments connecting rich galaxy clusters and forming a super-cluster structure, it is important to increase our knowledge of these sources.

The best known case is the poor group of galaxies $0217+70$, where an extended central radio halo and double peripheral relic radio sources are present (Brown et al. 2011). Further cases are the radio halo in A523 and in A1213 (Giovannini et al. 2009).

We present in Fig. 2-Left the plot of the total radio power and X-ray luminosity for radio halos as shown in Feretti et al. (2012), where X-ray underluminous clusters are indicated by their name. These halos are overluminous in radio by at least an order of magnitude with respect to the expectation of the radio power - X-ray luminosity correlation. This new population of diffuse radio halos emission opens up the possibility of probing the correlation between low-mass cluster mergers and non-thermal cluster properties (particle acceleration and diffuse magnetic fields) with upcoming deep radio continuum surveys.

\section{Two peculiar structures: $0917+75$ and A399-A401}

The radio source 0917+75 (Fig. 3) is an elongated diffuse emission studied by Dewdney et al. (1991); Harris et al. (1993), and Giovannini \& Feretti (2000). It is located in a region away from rich clusters, the nearest one being A786 $(\mathrm{z}=0.124)$ at $\sim 4 \mathrm{Mpc}$. This distance is approximately twice the A786 virial radius, too large for the radio emission being the result of present or past interaction with the cluster. In this region, a few galaxy clusters at the same redshift are present (A787, A762, A748, A765), which belong to the Rood Group of clusters of galaxies N. 27. Their very large distance from the source favours the hypothesis that the diffuse radio structure is related 

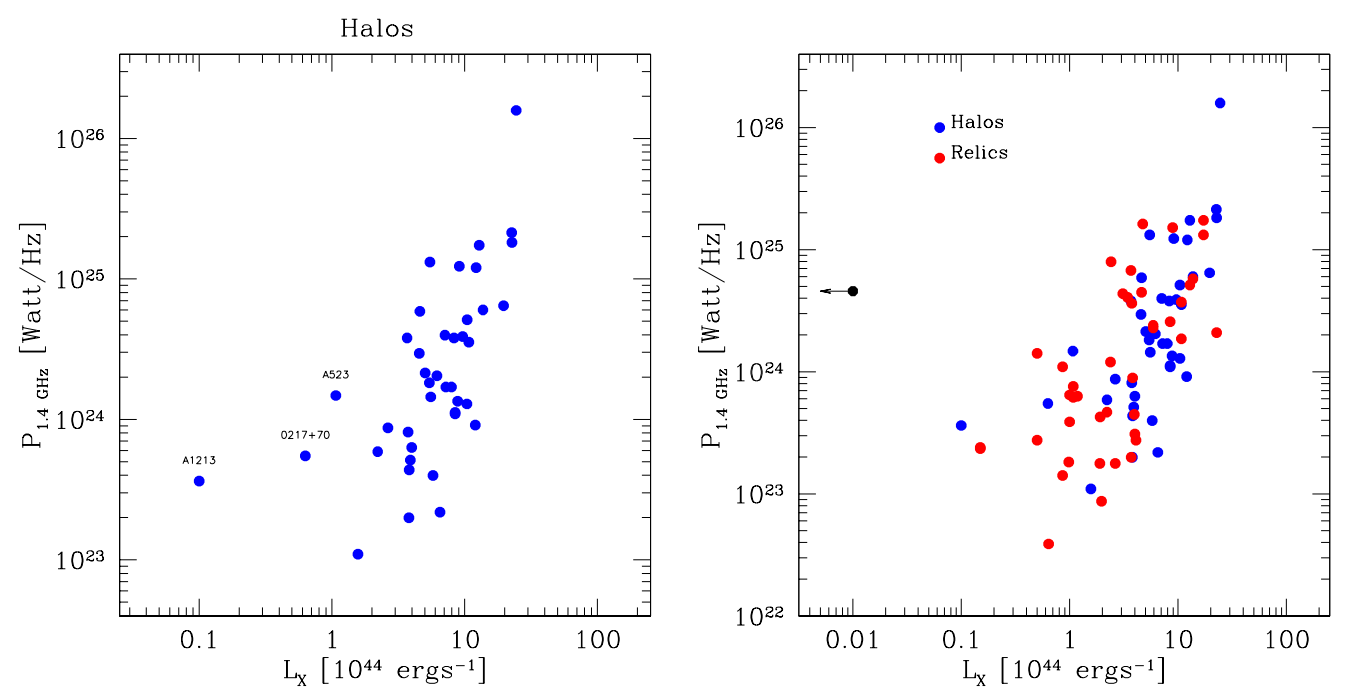

Figure 2: Left: monochromatic radio power of radio halos at $1.4 \mathrm{GHz}$ versus the cluster X-ray luminosity between 0.1 and $2.5 \mathrm{keV}$ for merging clusters with radio halos. Underluminous X-ray clusters are named (from Feretti et al. 2012). Right: monochromatic radio power of radio halos (blue) and relics (red) at 1.4 $\mathrm{GHz}$ versus the cluster X-ray luminosity between 0.1 and $2.5 \mathrm{keV}$ for merging clusters (data are from Feretti et al. 2012). The peculiar source $0917+75$ is shown in black.

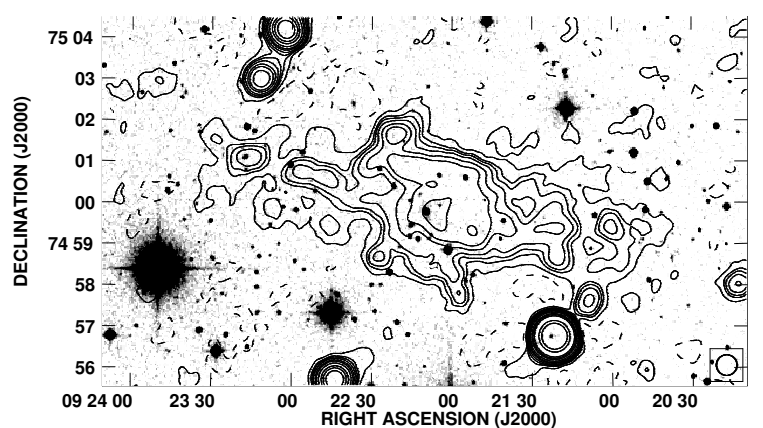

Figure 3: Isocontour image of the diffuse source $0917+75$ at $1.4 \mathrm{GHz}$ and HPBW $=20$ ". Contour levels are: $-0.20 .20 .50 .711 .52357103050 \mathrm{mJy} /$ beam, overimposed on the optical image from the DPSS.

to a galaxy group or to the supercluster structure. Dewdney et al. (1991) noted that a group of galaxies with an apparent magnitude similar to that of galaxies in A786 is present in the region of the diffuse radio emission and obtained the redshift of the two brightest galaxies $(0.1241$ and 0.1259 ) confirming the association. Girardi et al. (in progress) studied the distribution of galaxies of the surrounding large scale structure using the SuperCOSMOS Sky Survey. The distribution of the photometric members of the supercluster shows the close clusters as clear overdensities, while several faint galaxies are present in the $0917+75$ region, around the two brightest galaxies observed by Dewdney et al. (1991), confirming the presence of a group or a filament. The total spectral index is complex as discussed by Harris et al. (1993), showing an average value between $150 \mathrm{MHz}$ and $1.5 \mathrm{GHz}$ of $\sim 1.5$, with a possible high frequency steepening. The source is polarized at a $25 \%$ level at $1.4 \mathrm{GHz}$ in the brightest inner region (Giovannini et al., in preparation), unexpectedly high for a radio halo. 

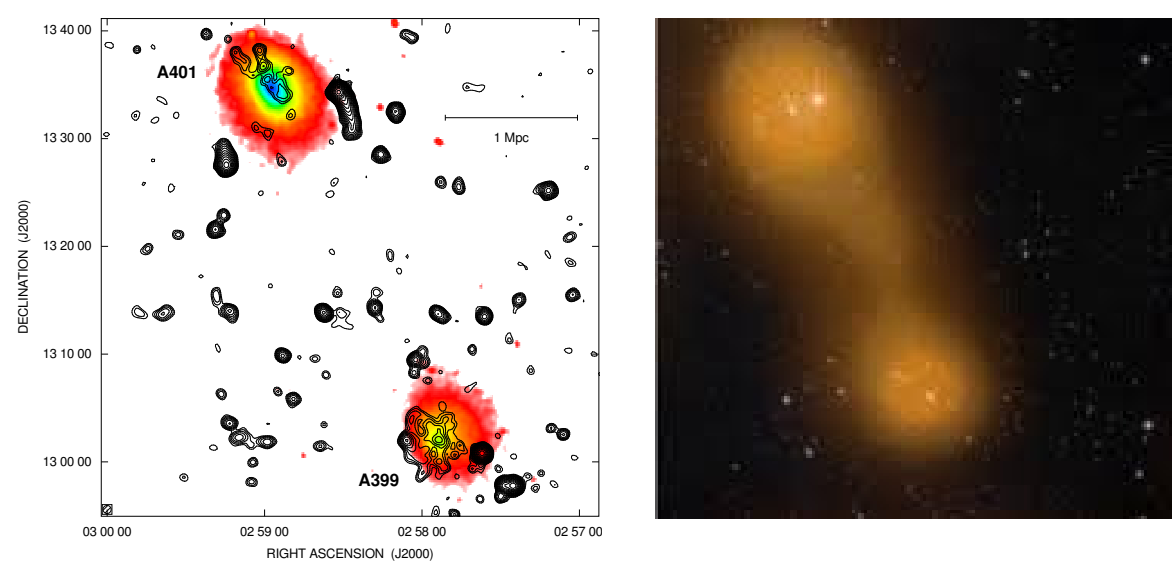

Figure 4: Left: Total intensity radio contours at 1.4 GHz of the system A399-A401 (Murgia et al. 2010), overlaid on the XMM X-ray image. Right: Sunyaev-Zeldovich emission (Planck collaboration 2013) showing a bridge of hot gas connecting A399-A401.

The main peculiarity of this source is the lack of X-ray emission (see Fig. 2-Right). This is unexpected in comparison with other Mpc scale diffuse cluster radio sources (halos or relics), where the radio power is correlated to the X-ray luminosity. X-ray upper limits obtained with the XMMNewton observatory, impose upper limits on the X-ray flux due to inverse Compton scattering of photons from the cosmic microwave background by relativistic electrons in the diffuse source and imply that the local magnetic field has to be $>0.81 \mu$ Gauss (three sigma level, Chen et al. 2008). This makes this source quite unique. We suggest that this source could be an inherent feature of the filamentary structure of the Rood 27 supercluster, even if the measured magnetic field lower limit is higher than expected in filamentary structures according to numerical simulations (see e.g. Ryu et al. 2008). Alternatively it could be the remnant of a died radio galaxy. If confirmed it will suggest that AGN activity is important to spread seeds of relativistic particles and magnetic fields in filaments on a scale as large as $\sim 2 \mathrm{Mpc}$.

A399 and A401 is a pair of galaxy clusters separated in projection by an angular distance of 36', corresponding to a linear separation of $3 \mathrm{Mpc}$. It represents the first example of a double radio halo in the close pair of galaxy clusters (Murgia et al. 2010, Fig. 4). The discovery of this double halo is exceptional, owing to the rarity of halo radio sources in general, and given that X-ray data (e.g. Sakelliou \& Ponman 2004, Fujita et al. 2008), seem to suggest that the two clusters are still in a pre-merger state. The existence of these radio halos leads naturally to the question as to whether faint radio emission related to weaker magnetic fields could be present in the intergalactic medium between the two clusters, namely on the scale of galaxy filaments. This question is supported by new Planck satellite data that revealed a Sunyaev-Zeldovich (SZ) signal between A399 and A401 (Planck Collaboration, 2013). The intercluster SZ signal is compatible with a scenario where the intercluster region is populated with a mixture of material from the clusters and the intergalactic medium, indicating the presence of a bridge of matter connecting the two systems. It may be an evidence that the region is witnessing the process of a large-scale structure formation, where cosmic shocks originated by complex merger events are able to amplify magnetic fields and accelerate synchrotron electrons. 


\section{The Future}

To improve our knowledge on the origin and properties of Mpc scale magnetic fields we need a comprehensive view of the radio emission on very large scales, to complement the information obtained from the well known halos and relics present in high X-ray luminous merging clusters:

1) non-thermal emission in larger structures, going from radio halos in clusters to the radio bridges connecting clusters to galaxy filaments and superclusters;

2) non-thermal emission from galaxy clusters with low mass and low X-ray luminosity, often present in supercluster structures connecting rich clusters.

From the surface brightness in the few galaxy filaments discussed above, we can estimate the needs of the expected new observations with SKA1 for a deeper understanding of large scale magnetic fields. The two prototypes of these sources are considered to be $\mathrm{ZwCl} 2341.1+0000$ and the bridge in the Coma cluster. In $\mathrm{ZwCl} 2341.1+0000$, the surface brightness in fainter diffuse regions is of the order of $0.05 \mu \mathrm{Jy} / \operatorname{arcsec}^{2}$ at $1.4 \mathrm{GHz}$. The bridge of radio emission connecting the Coma-C radio halo with the Coma relic source $1253+275$ has been detected only at $327 \mathrm{MHz}$ (Giovannini et al. 1990) and its surface brightness $\sim 0.02 \mu \mathrm{Jy} / \operatorname{arcsec}^{2}$ at $1.4 \mathrm{GHz}$, assuming a spectral index of 1.5 between 327 and $1415 \mathrm{MHz}$. From these data we estimate that a sensitivity of $\sim 10^{-2} \mu \mathrm{Jy} / \operatorname{arcsec}^{2}$ at $1.4 \mathrm{GHz}$ are needed. In addition, high angular resolution is necessary to avoid confusion limited images. The required compromise between high surface brightness sensitivity and high resolution is best achieved with SKA1-MID (with respect to SKA1-SUR). Comparing Key System Performance Factors for SKA1-MID Array (Table 6 and 7 in Dewdney et al. 2013), with the confusion level estimated by Condon et al. (2012), the best choice would be to observe in Band 2 with the maximum available bandwidth $(0.8 \mathrm{GHz})$ and an angular resolution of $3^{\prime \prime}$. The minimum detectable flux density in a 2 hour observation should be $\sim 0.08 \mu \mathrm{Jy} / \mathrm{beam}$, at the same level of the noise-free confusion limit $=0.08 \mu \mathrm{Jy}$ at this angular resolution. Since the regions with the lowest surface brightness (assuming the conservative hypothesis of uniform distribution) in our sources are at a level of $0.5-0.2 \mu \mathrm{Jy} / \mathrm{beam}$, suggested observations should be able to detect likely at 2.5-6 sigma level these kind of radio structures in all covered sky.

To investigate the chance to detect faint halos, we used the synthetic radio halos generated by $\mathrm{Xu}$ et al. (2012), under the assumption that the initial magnetic fields are injected into the ICM by AGN at high redshift. Assuming energy equipartition between magnetic fields and non-thermal electrons, Xu et al. compared the global properties of the mock radio halos with the real ones (see Fig. 5). The synthetic radio halos agree with the observed correlations between the radio power versus the cluster X-ray luminosity and between the radio power versus the radio halo size. Out of 16 simulated clusters, eight present a radio halo detectable by present radio telescopes (e.g. JVLA) under the hypothesis of equipartition condition. The eight simulated clusters whose radio emission is below the detection threshold will need deeper radio observations. Using upper limit on total radio power $\mathrm{P}_{1.4 \mathrm{GHz}}$ for the undetected radio halos, assuming a putative size as derived from the total radio power versus the largest linear size correlation, and an average surface brightness of the diffuse emission uniform over the putative halo size, the undetected clusters should host at $\mathrm{z}=0.2$ radio halos with a $1.4 \mathrm{GHz}$ surface brightness $\sim 0.3 \mu \mathrm{Jy} / \mathrm{beam}$, assuming a 3" HPBW. Therefore, also in this case, SKA1-MID observations in 2 hours should be able to detect at 4 sigma level these faint radio halos up to at least $\mathrm{z}=0.2$ (see Fig. 5). 

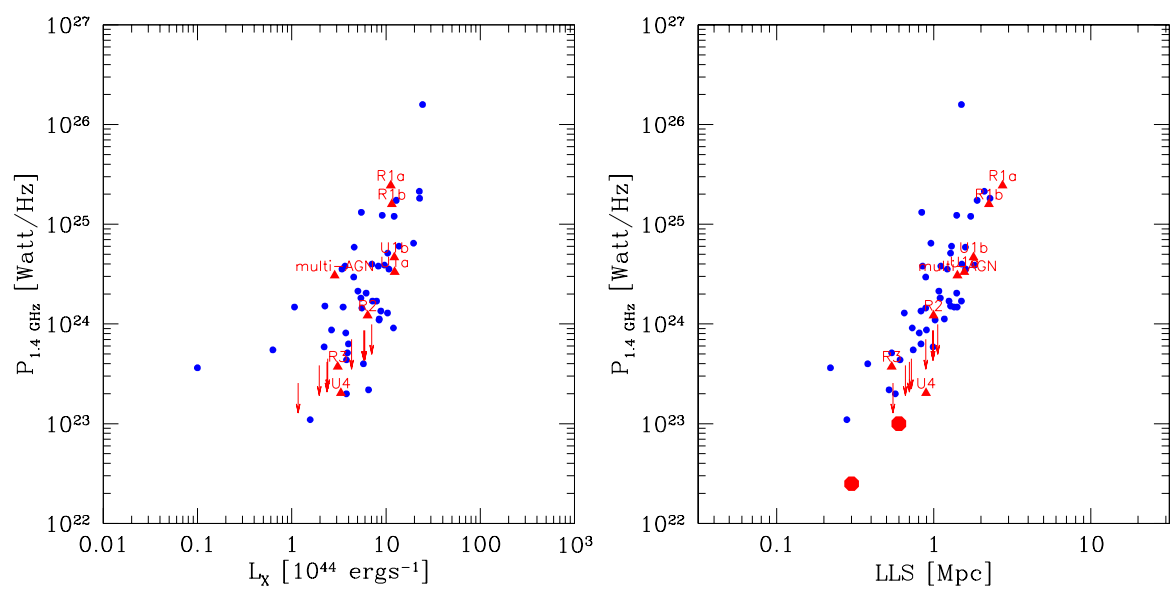

Figure 5: Left: Radio power of radio halos at $1.4 \mathrm{GHz}$ versus the cluster X-ray luminosity in the $0.1-2.4$ $\mathrm{keV}$ band. Right: radio power of halos at $1.4 \mathrm{GHz}$ versus their largest angular size (LLS). Blue dots are observed clusters, red triangles are simulated clusters, while arrows indicate upper limits on the radio power of simulated clusters (from Xu et al. 2013). Red circles on the Right panel are radio halos detectable at 4 sigma level with SKA1-MID Band 2 at $\mathrm{z}=0.2$, as discussed in the text.

A caveat is that the angular size of radio emission connected to galaxy filaments is unknown. Moreover, low power sources are best detected at low redshift because of higher flux density, but they could show a large angular size. We note that the bridge of emission in the Coma cluster, as well as the Coma-C radio halo, are not detected by JVLA at $1.4 \mathrm{GHz}$, not because of sensitivity limit, but because of missing short spacings (the JVLA shortest baseline is $\sim 40$ meters). To study this kind of structures at this frequency, SKA1-MID should have baselines shorter than $20 \mathrm{~m}$. Observations at low frequencies (SKA1-MID in Band-1, e.g. $700 \mathrm{MHz}$, and SKA1-LOW) are very suitable to detect this diffuse, steep spectrum radio emission. However, a resolution of a few arcsec is required to avoid confusion limit problems. With a maximun baseline of $100 \mathrm{~km}$, a HPBW $\leq$ 5 " is reached down to $150 \mathrm{MHz}$, thus allowing these observations. The SKA1-LOW requested sensitivity is $\sim 10 \mu \mathrm{Jy} / \mathrm{beam}$.

At higher frequencies (i.e. SKA1-MID, Band 3 or 4), these sources are not detected because of missing short spacings, therefore a combination with single-dish data is necessary.

In conclusion, with these observing specifications we expect to be able to estimate the magnetic field strength and radial profile both from synchrotron emission and from Faraday Rotation measure of diffuse and discrete sources within or behind clusters.

Moreover, the large bandwidth will allow to study with a single observation the spectral index distribution of diffuse sources to derive the origin and properties of relativistic particles and acceleration mechanism. Comparison with X-ray images will permit to individuate reacceleration regions and the presence of shocks in the intra-cluster medium. 


\section{References}

Bagchi, J., Enßlin, T. A., Miniati, F., et al., 2002, New Astronomy, 7, 249

Bonafede, A., Feretti, L., Murgia, M., et al.: A\&A 513, 30

Bonafede, A., Vazza, F., Brüggen, M., et al.: 2013 MNRAS 433, 3208

Boschin, W., Girardi, M., Barrena, R.: 2013 MNRAS 434, 772

Brown, S. \& Rudnick, L.: 2009 AJ 137, 3158

Brown, S. \& Rudnick, L.: 2011 MNRAS 412, 2

Brown, S., Duesterhoeft, J., Rudnick, 2011, ApJ 727, L25

Brunetti, G. \& Lazarian, A.: 2011 MNRAS 412, 817

Cassano, R., Ettori, S., Giacintucci, S., et al.,: 2010 ApJ, 721, 82

Chen, C.M.H., Harris, D.E., Harrison, F.A., et al., 2008 MNRAS 383, 1259

Condon, J.J., Cotton, W.D., Fomalont, E.B., et al. 2012 ApJ 758, 23

Dewdney, P.E., Costain, C.H., McHardy, I., et al. 1991 ApJS 76, 1055

Dewdney, P.E., Turner, W., Millenaar, R., et al. 2013 SKA1 System Baseline Design SKA-TELSKO-DD-001

Feretti, L., Giovannini, G., Govoni, F., Murgia, M.: 2012, Astron. \& Astrophys. Rev. 20, 54

Fujita, Y., Tawa, N., Hayashida, K., et al.: 2008 PASJ 60, 343

Giovannini, G., \& Feretti, L.: 2000 New Astronomy, 5, 335

Giovannini, G., Kim, K. T., Kronberg, P. P., Venturi, T.: 1990 IAUS 140, Edrs. R. Beck, P.P. Kronberg, R. Wielebinski p.492

Giovannini, G., Bonafede, A., Feretti, L., et al.: 2009 A\&A, 507, 1257

Giovannini, G., Bonafede, A., Feretti, L., et al., 2010 A\&A, 511, L5

Giovannini, G., Feretti, L., Girardi, M., et al.: 2011 A\&A, 530, 5

Giovannini, G., Vacca, V., Girardi, M., Feretti, L., et al. 2013 MNRAS 435, 518

Harris, D. E., Stern, C. P., Willis, A. G., et al.: 1993 AJ, 105, 769

Kim, K.-T., Kronberg, P. P., Giovannini, G., et al., Nature, 341, 720 (1989)

Murgia, M., Govoni, F., Feretti, L., Giovannini, G.: 2010 A\&A 509, 86

Ryu, D., Kang, H., Cho, J., Das, S.: 2008 Science 320, 909

Sakelliou, I., \& Ponman, T. 2004 MNRAS 351, 1439

Planck collaboration VIII, 2013 A\&A 550, 134

Vacca, V., Murgia, M., Govoni, F., Feretti, L., et al. 2012 A\&A 540, 38

Vazza F., Brunetti, G., Gheller, C., Brunino, R.: 2010 NewA 15, 695

Vazza F., Ferrari C., Bonafede A., et al.: 2015 "Filaments of the radio cosmic web: opportunities and challenges for SKA", in proc. Advancing Astrophysics with the Square Kilometer Array, PoS(AASKA14)097

Xu, H., Govoni, F., Murgia, M., et al.: 2012 ApJ 759, 40 